Military Technical College Kobry El-Kobbah, Cairo, Egypt

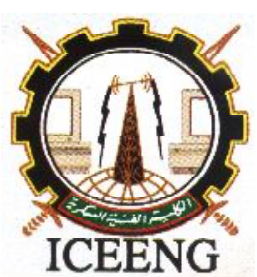

\author{
$10^{\text {th }}$ International Conference \\ on Electrical Engineering \\ ICEENG 2016
}

\title{
Voltage Responsivity Measurements for PANI-DBSA/PVDF Infrared Sensor Using Electrical Chopped Circuit
}

\author{
By
}

\begin{abstract}
Mazhar B. Tayel ${ }^{1}$, Moataz B. Soliman ${ }^{2}$ Shaker M. Ebrahim ${ }^{2}$ and Ahmed M. El-Shaer ${ }^{1 *}$
${ }^{1}$ Department of Electrical Engineering, Faculty of Engineering, Alexandria University, lofty Elsied, Alexandria, Egypt, 11432.

${ }^{2}$ Materials Science Department, Institute of Graduate Studies and Research, Alexandria University, 163 Horrya, Alexandria, Egypt, 21526.
\end{abstract}

\section{Abstract:}

The pyroelectrical response of infrared sensor dodycylbenzene sulfonic acid (DBSA) doped polyaniline (PANI) and polyvinylidene fluoride (PVDF) blend films was reported in this work. Modulation frequency dependence of the pyroelectric response of PANIDBSA/PVDF blend films with different weight percentages $(0,10$, and 20) of PANI has been investigated using the proposed an electrical chopped circuit. The electrical chopped circuit is composed of computerized Arduino board to generate the chopped frequency and shielded with the transmitting circuit, infrared sensor blend film, and the amplification circuit. The PANI-DBSA/PVDF blend film with 10 wt.\% of PANI showed the optimum the pyroelectric response. The voltage responsivity (Rv) of PANIDBSA/PVDF with $10 \mathrm{wt} . \%$ of PANI was $158 \mathrm{~V} / \mathrm{W}$ at $0.2 \mathrm{~Hz}$ and the noise equivalent power (NEP) of was $2.8 \times 10^{-7} \mathrm{~W} / \mathrm{Hz}^{1 / 2}$ at $100 \mathrm{~Hz}$. The results indicated that the PANIDBSA/PVDF blend film with $10 \mathrm{wt} . \%$ of PANI are suitable as an active material for pyroelectric infrared sensor.

\section{Keywords:}

Pyroelectric, Infrared Sensor, Polyaniline, Polyvinylidene fluoride, Voltage responsivity.

* Corresponding to Ahmed Mohamed El-Shaer,

Department of Electrical Engineering, Faculty of Engineering, Alexandria University, Alexandria, Egypt,

Tel.+201220600050, E-mail: ahshaer@alexu.edu.eg, ahshaer1@yahoo.com. 


\section{Introduction:}

Infrared radiation is radiated from any object in the form of heat. The sources of energy that create low temperatures without producing visible light generate infrared energy, for example; humans, animals, and objects. So, everything on the earth has infrared (IR) energy. The IR energy of an object depends on different parameters such as its temperature, colour, and texture. This energy is not visible with human eye, but pyroelectric infrared (PIR) sensors can detect it [1]. Pyroelectric IR sensors can distinguish changes in temperature due to incident electromagnetic radiation. The sensor will produce an electric charge in response to the changes in temperature of the pyroelectric material. This characteristic allows the use of this sensor in many applications such as motion sensor, security systems, automatic doors, and automatic light control because of its low power consumption and the simplicity of wiring the signal processing circuitry of the subsequent stage [2]. The choice of the pyroelectric material is mainly determined by (a) its responsivity and noise equivalent power, (b) availability and durability of pyroelectric material, (c) environment in which the material has to operate, and (d) the purpose for which the sensor is employed [3]. The material most used in pyroelectric IR sensor had the form of single crystal and ceramic. Most commercial sensors are based on ceramic materials because of their high pyroelectric constant. In order to improve the responsivity, one should decrease the heat capacity and thermal conductivity of the pyroelectric materials. Recently, polymeric films such as polyvinylidene fluoride (PVDF) or polyaniline (PANI) have come into increasing use as sensor elements in pyroelectric devices, because their small heat capacity and low thermal conductivity compared to ceramics [4]. However, the pyroelectric coefficient is too low compared to ceramic materials. In contrast, the ceramic/polymer composite materials can combine the pyroelectric activities of the ceramic and the formability and low thermal conductivity of the polymer. These advantages of the composite materials make them very sensitive to the temperature change [5].

In this work, we aim to prepare PANI-DBSA/PVDF blend films with different weight percentages of PANI. The pyroelectric parameters of Rv and NEP of the PANIDBSA/PVDF blend were measured and investigated using the proposed and homemade electrical chopper circuit.

\section{Experimental Work:}

\subsection{Pteparation of Polyaniline:}

The preparation steps of PANI are illustrated in Figure 1. PANI in the doped form was prepared by chemical oxidative polymerization with hydrogen peroxide $\left(\mathrm{H}_{2} \mathrm{O}_{2}\right)$ as an oxidant. Typically $6.57 \mathrm{ml}$ of aniline was dissolved in $900 \mathrm{ml}$ hydrochloric acid, and then $0.02 \mathrm{~g}$ of ferrous chloride $\left(\mathrm{FeCl}_{2}\right)$ was dissolved in $225 \mathrm{ml}$ deionized water. The two solutions were then mixed and stirred for $48 \mathrm{hr}$. During the stirring $100 \mathrm{ml}$ of $\mathrm{H}_{2} \mathrm{O}_{2}$ 
was drop wise to the solution to initiate the polymerization. The green powder of PANI was collected on a filter paper using vacuum filtration and washed with water until filtrate becomes colourless, then dried at $50{ }^{\circ} \mathrm{C}$ for $24 \mathrm{hr}$ [6]. The un-doped PANI was obtained with ammonia solution and stirred for $3 \mathrm{hr}$. The blue powder was collected on a filter paper using vacuum filtration and washed with water until filtrate becomes neutral, then dried at $50^{\circ} \mathrm{C}$ for $24 \mathrm{hr}$.

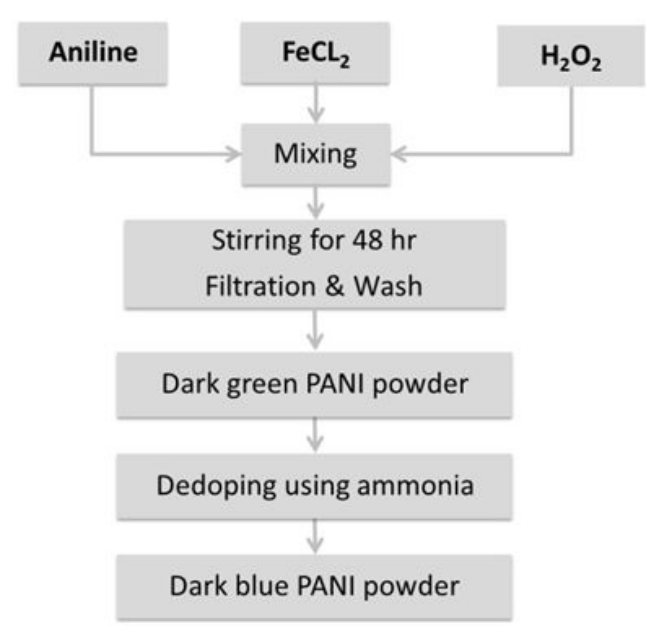

Figure (1): Preparation steps of PANI

\subsection{Preparation of PANI-DBSA/PVDF Blend Films}

Doping the PANI was performed by dissolving $0.5 \mathrm{~g}$ of PANI in $20 \mathrm{ml}$ of $\mathrm{N}, \mathrm{N}$ Dimethylformamide (DMF) and stirring for $48 \mathrm{hr}$. The blue solution of freshly distilled aniline and $0.175 \mathrm{gm}$ of (DBSA) was added in $50 \mathrm{ml}$ flask and stirred for $12 \mathrm{hr}$ to obtain a PANI-DBSA composite. Two gram of PVDF was dissolved in $20 \mathrm{ml}$ of DMF. The weight percentages of the prepared blend films with thickness were tabulated in Table 1. The prepared solutions were stirred for $1 \mathrm{hr}$ and casted on the petri dish to obtain the blend films at $80^{\circ} \mathrm{C}$ for $1 \mathrm{hr}$.

Table 1: Weight percentage of PANI-DBSA/PVDF blend films with its thickness

\begin{tabular}{|c|c|c|c|}
\hline \multicolumn{4}{|c|}{ PANI-DBSA/PVDF Blend Films } \\
\hline Weight percentage of PANI-DBSA & $\begin{array}{c}\text { PANI-DBSA } \\
\text { content (ml) }\end{array}$ & $\begin{array}{c}\text { PVDF } \\
\text { content (ml) }\end{array}$ & Thickness ( m) \\
\hline $0 \mathrm{wt} . \%$ & $0 \mathrm{ml}$ & $2 \mathrm{ml}$ & $32 \mathrm{~m}$ \\
\hline $10 \mathrm{wt} \%$ & $2.81 \mathrm{ml}$ & $2 \mathrm{ml}$ & $55 \mathrm{~m}$ \\
\hline $20 \mathrm{wt} . \%$ & $8.52 \mathrm{ml}$ & $2 \mathrm{ml}$ & $80 \mathrm{~m}$ \\
\hline
\end{tabular}




\section{Design PIR Sensor measurement Circuit Based Electrical Chopper}

The pyroelectric IR sensor is sensitive to the variation of temperature and its response is related to the chopped frequency, thus the investigation of the frequency dependence pyroelectric property is critical. The incident infrared was periodically chopped for the ordinary pyroelectric sensor in order to generate a continuous output voltage or current. However, the chopping system is large in size, requires high power, and shows low reliability [40]. Figure 2 shows the schematic circuit diagram of electrical chopper based on Arduino UNO board. The first part consists of an Arduino UNO board that can be programmed with $\mathrm{C}$ language computer programming via Arduino 1.0.5 compiler to generate a square wave with modulation frequency in the range of chopper frequency $(0.01 \mathrm{~Hz}$ to $100 \mathrm{~Hz})$ [3-4]. The generated electric pulsed is converted to free air infrared signal using infrared emitting diode as a transmitter has power of $25 \mathrm{~mW}$. The second part of Figure 2, includes both sensing element which coated with a silver paste in both sides and the current to voltage circuit based on op-amp LF351. The pyroelectric sensing element was mounted inside a window of two point probe holder fabricated on printed circuit board. The holder has two output terminals, one of them was connected to the ground and the other terminal was connected as an input to detecting circuit.

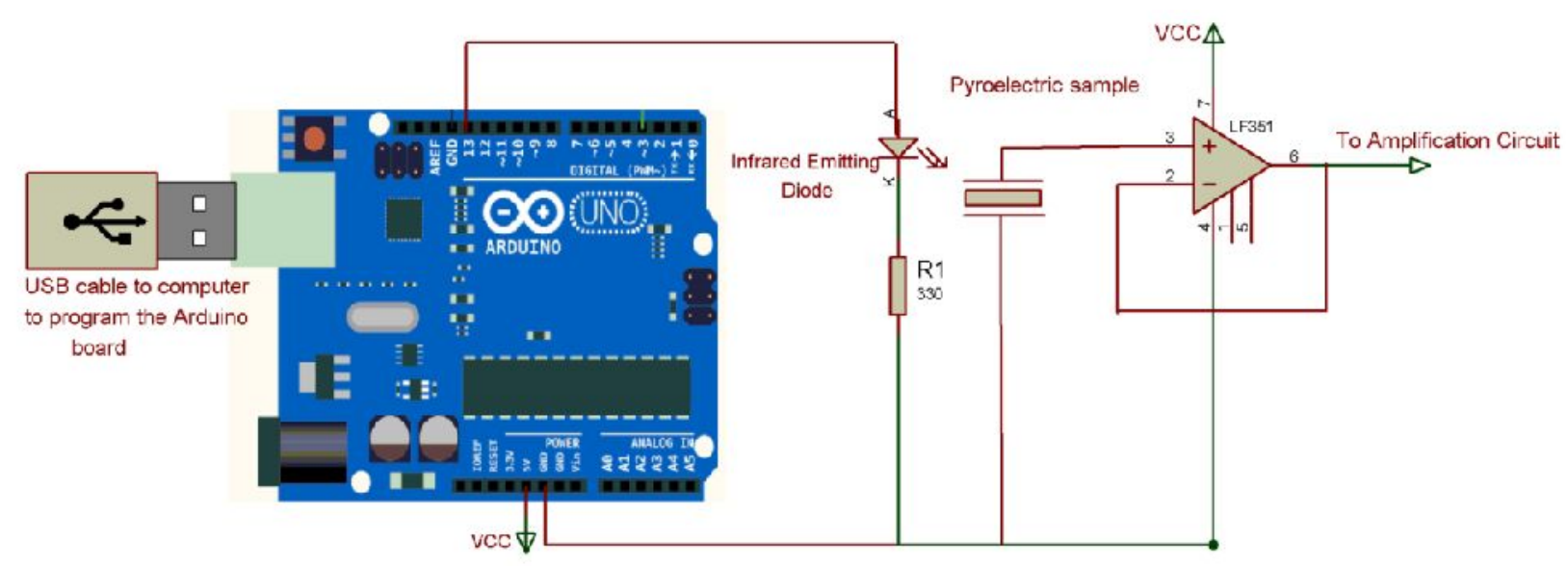

Figure (2): PIR sensor measurement circuit with electrical chopper frequency.

The output signal voltage from the operational amplifier (LF351) is in the range of 1 $\mathrm{mVpp}$. This small voltage is around a DC signal that may significantly vary from on sensor to another. For this reason, it should be to cancel the DC part of the signal and to amplify the AC part. As the signal was distributed by the environment, a noise filter was useful. Figure 3 presents the three-stage architecture based on operational amplifier that used to amplify the voltage signal of a pyroelectric sensor. 


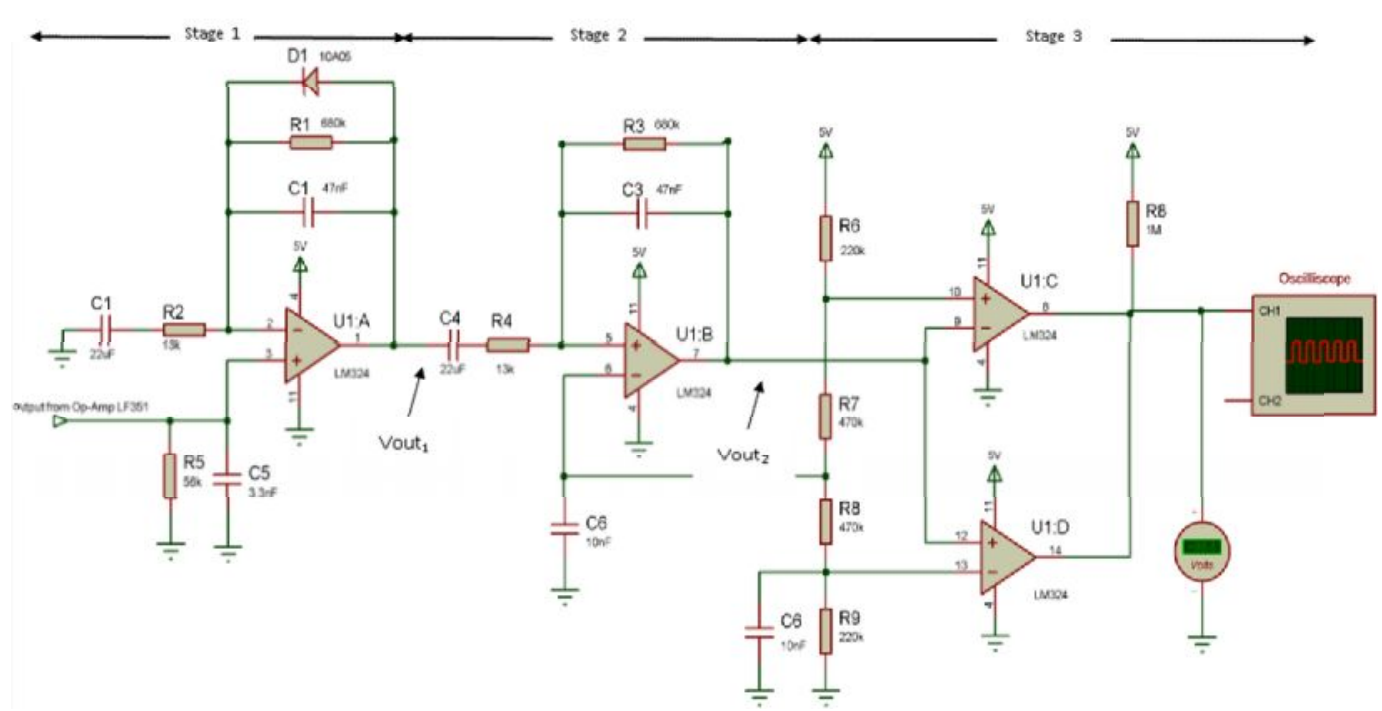

Figure (3): Global schematic showing all architectural stages used to amplifier the detecting signal [3].

The first stage as shown in Figure 3 amplifies the voltage signal. It cancels the DC part of the signal and filters the high frequency noise that could lead to false detections. As shown in stage 1, the stage gain is 53.3 (Gain $1=1+\mathrm{R} 1 / \mathrm{R} 2$ ). This amplification allows a usable signal that is higher than the noise level. The second stage is similar to stage 1 . It was used to filter and amplify the AC signal. The gain of this stage was 52.3 (Gain2=R3/R4). This gain means that after stage 2 the signal will amplify 2790 times. In stage 2, the Op-Amp common mode voltage was set by the divider bridge composed of R6, R7, R8, and R9. This allows greater amplification of the AC signal. In the third stage, when the IR signal is detected, the output of the Op-Amp U1:C and U1:D is in low state. The divider bridge composed of the resistor R6, R7, R8, and R9 was used to set the voltage reference of these devices. The LMF324 Op-Amp was an input/output single rail Op-Amp, and there is no constraint on the input common mode voltage. Thus, while the voltage references of U1:C and U1:D were within the Vcc range, the maximum output voltage for $\mathrm{U} 1: \mathrm{C}$ was set to $0.84 \times \mathrm{Vcc}$ [3]. When the voltage signal Vout 2 from stage 2 was above this reference, the output of U1:C was in low stat, and close to ground. U1:D was used to detect when the signal was below a certain reference. The voltage reference was set to $530 \mathrm{mV}$ thanks to the divider bridge composed of R6, $\mathrm{R} 7, \mathrm{R} 8$, and R9. The minimum output voltage was equal to $0.16 \times \mathrm{Vcc}$ according to [3]. After the signal output from the pyroelectric sensor was amplified with amplification circuit shown in Figure 3, the voltage responsivity was recorded using an oscilloscope (HITACHI digital storage VC-2060) or voltammeter. The measured voltage signal as a function of input frequency was used to calculate the voltage responsivity and noise equivalent power. Figure 4 shows the pyroelectric IR sensor measurement circuit as a shield with the Arduino UNO board. 


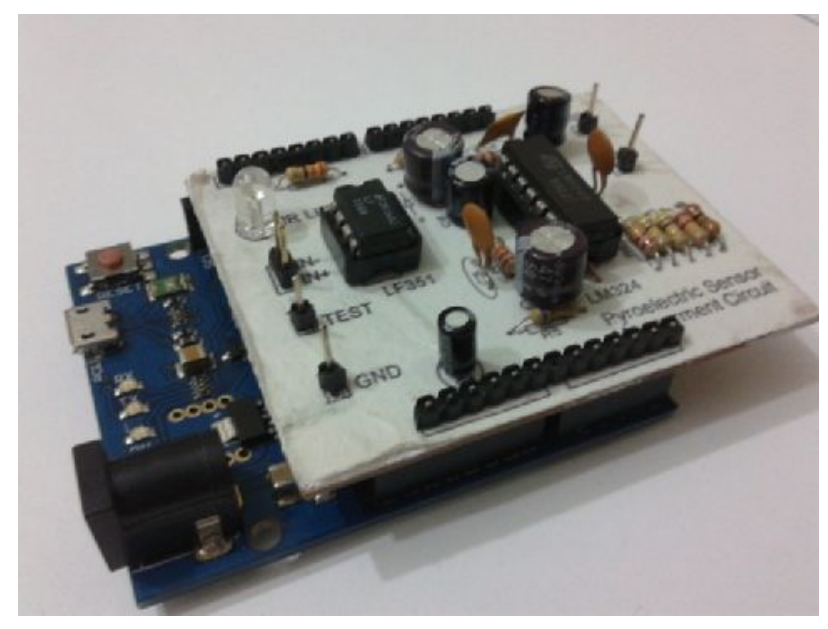

Figure (4): A prototype of pyroelectric IR sensor measurement circuit shielded Arduino UNO board.

\section{Result and Discussion:}

The IR response of the PANI-DBSA/PVDF blend films was measured using the proposed electrical chopped circuit. The voltage responsivity and noise equivalent power were calculated to evaluate fabricated infrared sensor. The IR response voltage is caused by the temperature oscillation in the specimen due to the incident chopped frequency.

\subsection{Voltage Responsivity of PANI-DBSA/PVDF Blend Films:}

Voltage responsivity is determined as a ratio of the voltage generated in the IR sensor $(\mathrm{V})$ to radiation power incident onto the sensor surface $\left(\mathrm{W}_{\mathrm{i}}=25 \mathrm{~mW}\right)$ :

$$
R_{V}=\frac{\Delta V}{W_{i}}=\frac{\eta \omega A p R_{t}}{G_{T H}\left(1+\omega^{2} \tau_{T H}^{2}\right)^{1 / 2}\left(1+\omega^{2} \tau_{E}^{2}\right)^{1 / 2}}
$$

where $\eta$ is the emissivity, $p$ is the pyroelectric coefficient, $A$ is the sensor area, $R_{t}$ are the electrical and thermal resistance, respectively, is the modulation frequency, $\tau_{\mathrm{E}}$ is the electrical time constant, and $\tau_{\mathrm{TH}}$ is the thermal time constant [7-8]. The voltage responsivity of PANI-DBSA/PVDF blend films is increased gradually with increase of the modulation frequency and reaching a maximum at frequency equal to $1 / \tau_{\mathrm{E}}$. Then, it has a constant value till the frequency equals to $1 / \tau_{\mathrm{TH}}$. Beyond this frequency, the voltage responsivity is reduced gradually. This behaviour is due to the cycle of the chopped wave which becomes shorter than thermal response time. The infrared sensor needs a certain thermal response time to warm up. However, the period of chopped wave becomes shorted than the thermal response time constant of the sensor as the modulation frequency increases. This makes the infrared radiation heating cannot achieve balance until the next cycle. Therefore, the infrared sensor could not be fully 
warmed with the higher modulation frequency, and the responsivity got smaller and smaller [8].

Figure 5 shows the modulation frequency dependence of $\mathrm{Rv}$ for the PANIDBSA/PVDF blend films with various wt.\% of PANI. The voltage responsivity increases with increasing frequency for $\omega<1 / \tau_{\mathrm{E}}$ and decrease with increasing frequency for $\omega>1 / \tau_{\mathrm{TH}}$, the values $\tau_{\mathrm{E}}$ and $\tau_{\mathrm{TH}}$ are estimated from measurement of the rising and falling edges from the observed output waveforms as shown in Figure 5. This trend is consistent with that reported by many authors [9-10].

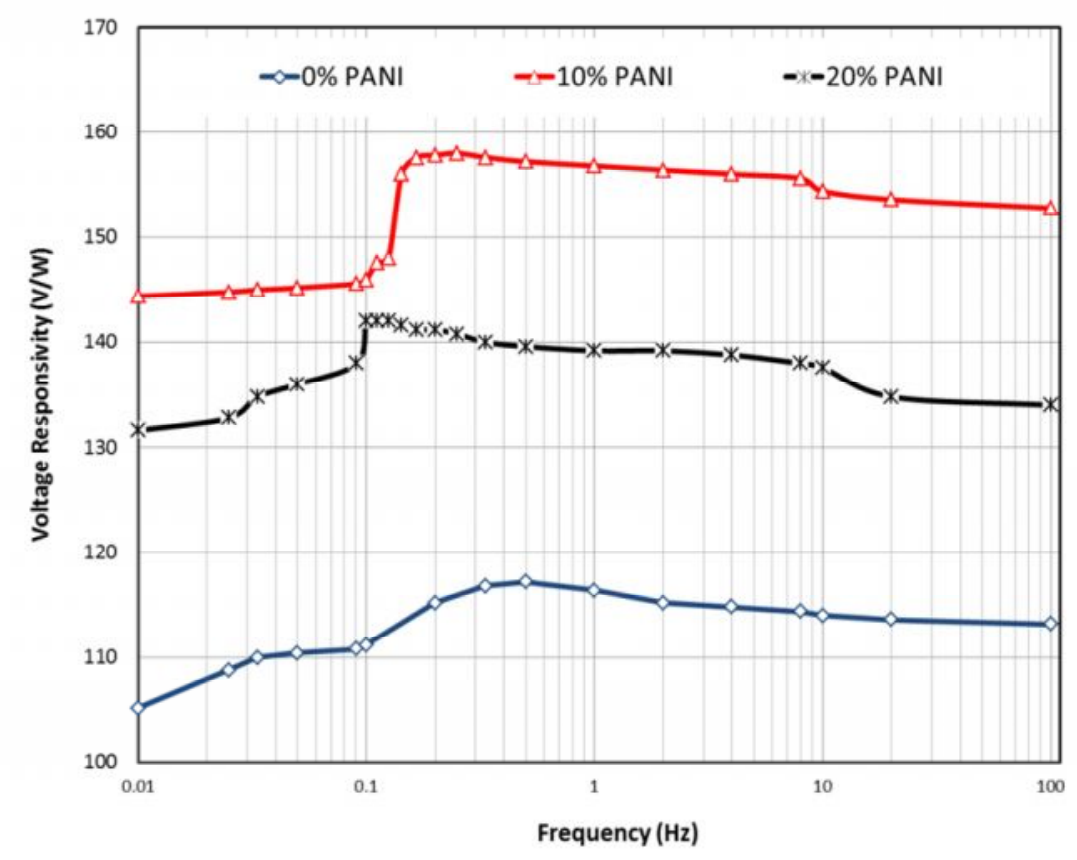

Figure (5): Voltage responsivity versus modulation frequency for PANI-DBSA/PVDF thin films with different wt.\% of PANI.

\subsection{Noise Equivalent Power of PANI-DBSA/PVDF Blend Films:}

In determining the sensor performance, it is insufficient to consider only its responsivity. But one has to consider various forms of unwanted signal called noise. The minimum detectable signal is limited by various noise sources in the sensor element in the load, and in the external current measuring circuit. The noise is expressed in unit $\mathrm{V} / \sqrt{\mathrm{Hz}}$. The sensitivity of a sensor is determined by the noise level in the amplified output signal. The noise level can be expressed in terms of the incident radiation on the sensor necessary to give a signal equivalent to the noise. If the noise voltage is $V_{N}$ then the noise equivalent power is defined as [13]:

$$
N E P=\frac{\Delta V_{N}}{R_{V}} \quad(W a t t / \sqrt{H z})
$$


The primary electronic noise sources for the circuit are the thermal or radiation noise $\mathrm{V}_{\mathrm{TH}}$, dielectric or Johnson noise $\mathrm{V}_{\mathrm{J}}$, amplifier current noise $\mathrm{V}_{\mathrm{I}}$, and amplifier voltage noise $\mathrm{V}_{\mathrm{V}}$. The total noise voltage is determined by summing the noise voltages $[4,13]$ :

$$
\Delta V_{N}^{2}=\Delta V_{T H}^{2}+\Delta V_{J}^{2}+\Delta V_{I}^{2}+\Delta V_{V}^{2}
$$

\subsubsection{Thermal Noise}

Thermal noise is the change in output voltage that arises from random changes in the temperature of the pyroelectric sensor. These thermal fluctuations are produced by the random exchange of heat and photons between the sensor and its surroundings. These thermal changes are consequence of incident radiation. The thermal noise, as a voltage is calculated by the expression below $[4,13]$ :

$$
\Delta V_{T H}=R_{V} \frac{P_{I}}{\eta^{1 / 2}}
$$

\subsubsection{Johnson Noise}

Johnson noise arises from the random motion of charge carriers in the crystal and in the electric circuit produces random fluctuations in voltage between its ends. The Johnson noise voltage is given by $[10,13]$ :

$$
\Delta V_{J}=\left(\frac{4 k T R}{1+\omega^{2} \tau_{E}^{2}}\right)^{1 / 2}
$$

\subsubsection{Amplifier Noise}

$\mathrm{V}_{\mathrm{I}}$ and $\mathrm{V}_{\mathrm{V}}$ are the noise produced by the electronic amplifier used in the detection system. The amplifier noise can be obtained from the manufacturer [14].

The values of noise voltage of the PANI-DBSA/PVDF thin film with various wt.\% of PANI are calculated. The modulation frequency dependence of the NEP of PANIDBSA/PVDF thin film with various wt.\% of PANI is shown in Figure 6. It is seen that NEP at low frequency is almost constant. These are due to the amplifier current $V_{I}$ and voltage $V_{V}$ noise [14]. As the frequency increases, the Johnson noise will be the dominant noise in the pyroelectric sensor and its value will be proportional to $\mathrm{f}^{-1 / 2}$. All the prepared samples have almost identical behaviour with frequency. A lower NEP indicates a better pyroelectric sensor. The PANI-DBSA/PVDF blend films of $10 \mathrm{wt} . \%$ of PANI exhibited the lowest NEP value which is equal to $2.8 \times 10^{-7} \mathrm{~W} / \mathrm{Hz}^{1 / 2}$ at $100 \mathrm{~Hz}$ as shown in Figure 6. 


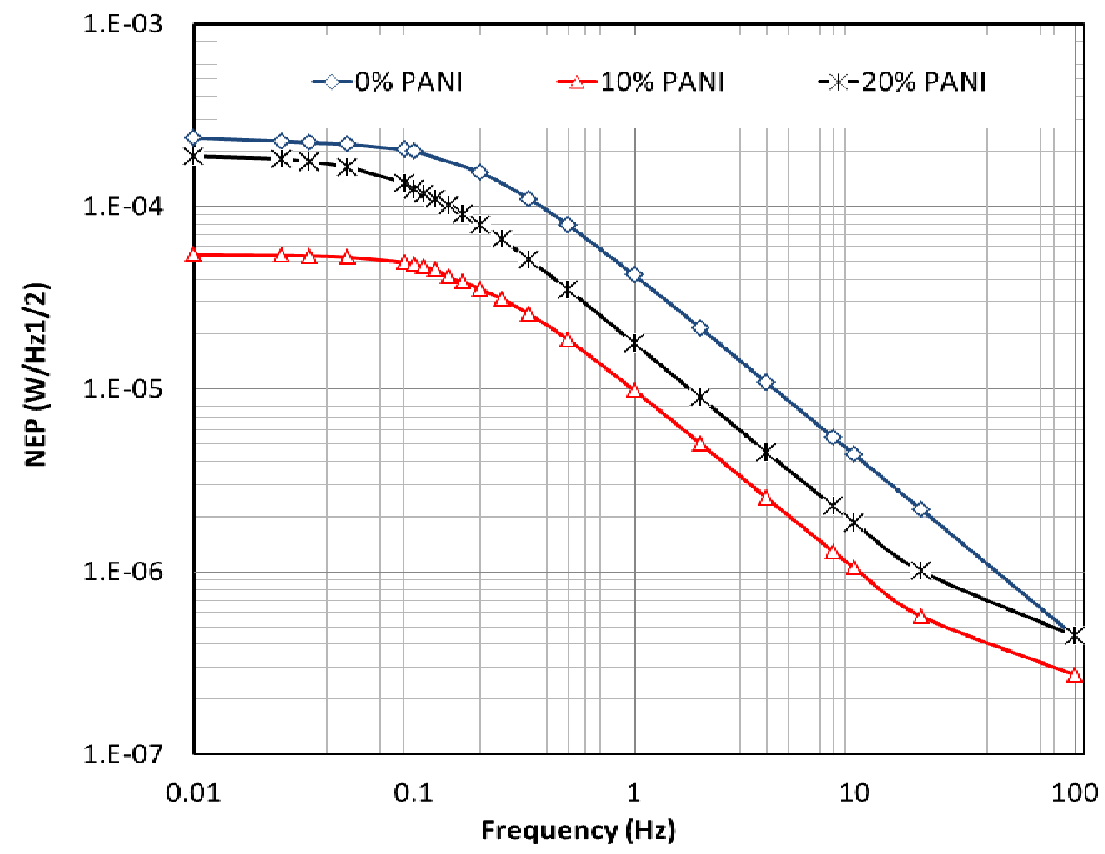

Figure (6): Noise equivalent power versus modulation frequency for PANI-DBSA/PVDF thin films with different wt.\% of PANI.

\section{Conclusions:}

We have successfully fabricated PANI-DBSA/PVDF blend films with PANI contents of 0,10 , and 20 wt. $\%$ by using chemical oxidative method, and the parameters of pyroelectricity as function of the PANI weight percentage were measured using the proposed homemade electrical chopped circuit. PANI-DBSA/PVDF blend film has 10 wt. $\%$ of PANI exhibited the largest voltage responsivity of $158 \mathrm{~V} / \mathrm{W}$ at $0.2 \mathrm{~Hz}$ and the noise equivalent power for the three blend films had a tendency to decrease with the square root of the modulation frequency due to the predominance of dielectric loss component in the Johnson noise. NEP indicated a minimum value of the incident energy in a pyroelectric IR sensor and the blend film has $10 \mathrm{wt} . \%$ of PANI exhibited a minimum value of $2.8 \times 10^{-7} \mathrm{~W} / \mathrm{Hz}^{1 / 2}$ at $100 \mathrm{~Hz}$.

\section{References:}

[1] E. B. Soyer, "Pyroelectric Infrared (PIR) Sensor Based Event Detection," M. Sci. thesis, Institute of Engineering and Sciences of Bilkent University, Ankara, Turkey, July. 2009.

[2] S. Masahiro, "Pyroelectric IR sensor with surface mount capability," Energy Devices Lett., vol. 7, no. 1 pp. 99-101, 2012.

[3] J. Fraden, Handbook of modern sensors physics, design, and applications, $3^{\text {rd }}$ ed., Imprint of Germany: Springer-Verlag, USA, 2003. 
[4] W. K. Sakamoto, S. T. Kagesawa, and W. B. Melo, "Voltage responsivity of pyroelectric sensor," Sensors and Actuators A: Physical, vol. 77, no. 1, pp. 28-33, 1999.

[5] C. G. Wu, P. Li, W. B. Luo, W. L. Zhang, "Quick response PZT/PVDF composite film pyroelectric infrared sensor with patterned polyimide thermal isolation method," Infrared Physics \& Technology, vol. 66, pp. 34-38, 2014.

[6] V. N. Bliznyuk, A. Baig, S. Singamaneni, and S. G. Shapoval, "Effects of surface and volume modification of polyvinylidene fluoride by polyaniline on the structure and electrical properties of their composites, Polymer, vol. 46, no. 25, pp. 1172811736, 2005.

[7] A. M. Elshaer, A. K. Aboulsoud, S. Ebrahim, M. Soliman, "Electrical chopped frequency circuit for characterizing pyroelectric sensor," Journal of Electrical Engineering, vol. 14, no. 4, pp. 1-6, 2014.

[8] A. Rogalski, "Quantum well photoconductors in infrared detector technology," Applied Physics, vol. 93, no. 8, pp. 4355-4391, 2003.

[9] B. Ploss, and S. Bauer, "Characterization of materials for integrated pyroelectric sensors," Sensors and Actuators A: Physical, vol. 26, no. 1, pp. 407-411, 1991.

[10] A. F. Armitage, K. Benjamin, D. Setiadi, H. Weller, and T. Binnie, "Infrared sensing using pyroelectric polymers," in Proc. of the Eighth Conference on Sensors and Their Applications, Glasgow, pp. 297-302. 1997

[11] A. M. Elshaer, M. B. Tayel, S. Ebrahim, M. Soliman, "Polyaniline/polyvinyledene Blends for pyroelectric sensor," in Proc. ICRPA-1, 1114 April, 2015, paper 076, p. 64.

[12] C. C. Chan, M. C. Kao, and Y. C. Chen, "Effects of membrane thickness on the pyroelectric properties of LiTaO3 thin film IR detectors," Japanese Journal of Applied Physics, vol. 44, no. 2, pp. 951-956, 2005.

[13] Z. Bielecki, and M. Brudnowski, "Method of popcorn-noise reduction," Optoelectronics Review, vol. 1, pp. 45-50, 2003.

[14] A. K. Batra, D. A. Aggarwal, and M. E. Edwards, "Present status of polymer: ceramic composites for pyroelectric infrared detectors," Ferroelectric, vol. 366, pp.84-121, 2008. 